\title{
A Novel Scientific Model for Rare and Often Neglected Neoplastic Conditions
}

\author{
Charles G. Fisher $^{1}$ Tony Goldschlager ${ }^{1} \quad$ Stefano Boriani ${ }^{2}$ Peter Pal Varga ${ }^{3} \quad$ Michael G. Fehlings $^{4}$
} Mark H. Bilsky ${ }^{5}$ Mark B. Dekutoski ${ }^{6}$ Alessandro Luzzati ${ }^{7}$ Richard P. Williams ${ }^{8}$ Sigurd Berven ${ }^{9}$ Dean Chou ${ }^{10}$ Jeremy J. Reynolds ${ }^{11}$ Nasir A. Quraishi ${ }^{12}$ Laurence D. Rhines ${ }^{13}$ Chetan Bettegowda ${ }^{14}$ Ziya L. Gokaslan ${ }^{14}$

1 Division of Spine, Department of Orthopaedics, University of British Columbia and Vancouver Coastal Health, Vancouver, British Columbia, Canada

${ }^{2}$ Department of Degenerative and Oncological Spine Surgery, Rizzoli Institute, Bologna, Italy

${ }^{3}$ National Center for Spinal Disorders and Buda Health Center, Budapest, Hungary

${ }^{4}$ Division of Neurosurgery, Department of Surgery, University of Toronto and Toronto Western Hospital, Toronto, Ontario, Canada

${ }^{5}$ Department of Neurosurgery, Memorial Sloan-Kettering Cancer Center, New York, New York, United States

${ }^{6}$ Orthopaedic Surgery, The CORE Institute, Sun City West, Arizona, United States

${ }^{7}$ Oncologia Ortopedica e Ricostruttiva del Rachide, Istituto Ortopedico Galeazzi, Milano, Italy

8 Department of Orthopaedics, Princess Alexandra Hospital, Brisbane, Queensland, Australia

${ }^{9}$ Department of Orthopaedic Surgery, University of California San Francisco, San Francisco, California, United States

10 Department of Neurological Surgery, University of California San Francisco, San Francisco, California, United States

${ }^{11}$ Spinal Division, Oxford University Hospital NHS Trust, Oxford, United Kingdom

${ }^{12}$ Queens Medical Centre, Nottingham University Hospital NHS Trust, Nottingham, United Kingdom

${ }^{13}$ Department of Neurosurgery, MD Anderson Cancer Center, The University of Texas, Houston, Texas, United States

${ }^{14}$ Department of Neurosurgery, Johns Hopkins University School of Medicine, Baltimore, Maryland, United States
Address for correspondence Charles Fisher, MD, MHSc, FRCSC, Blusson Spinal Cord Centre, 6th Floor - 818 W. 10th Avenue, Vancouver, BC, Canada V5Z1M9 (e-mail: charles.fisher@vch.ca).

Evid Based Spine Care J 2013;4:160-162.

\begin{abstract}
Study Type Retrospective cohort.

Introduction The treatment of rare neoplastic conditions is challenging, especially because studies providing high levels of evidence are often lacking. Such is the case

Keywords

- spine

- primary tumor

- scientific model

- rare disease

- collection with primary tumors of the spine (PTS), which have a low incidence, are pathologically heterogeneous, and have diverse treatment approaches. ${ }^{1}$ Despite these difficulties, appropriate evidence-based care of these complex patients is imperative. Failure to follow validated oncologic principles may lead to unnecessary mortality and profound morbidity. Objective With the aim of offering patients the most appropriate treatment based on the best available evidence, a novel scientific model was developed and employed. This article
\end{abstract}

received

June 9, 2013

accepted

July 18, 2013 (c) 2013 Georg Thieme Verlag KG

Stuttgart · New York
DOI http://dx.doi.org/ 10.1055/s-0033-1357365. ISSN 1663-7976. 
outlines this model, which has not only provided significant evidence guiding treatment of this rare condition, but we believe is readily transferrable to other similarly rare conditions.

Methods A four-stage approach was employed. (1) Planning: Data from large volume centers were reviewed together with results from a feasibility questionnaire to provide insight into epidemiology, patient volumes, tumor pathology, treatment modalities, and outcomes. (2) Recruitment: Centers with sufficient volume and valid data were enrolled and provided with the necessary infrastructure. This included study coordinators and a secure, Web-based database (REDCap, Vanderbilt University, Nashville, Tennessee, United States) to capture international data from six modules comprising: demographic, clinical, diagnostic, therapeutic, local recurrence, perioperative morbidity fields, and a cross-sectional survey to update survival information. The AOSpine Knowledge Forum Tumor designed these modules and provided funding. Each center received institutional ethics approval. (3) Retrospective stage: Prospectively collected data from all recruited centers were reviewed and analyzed retrospectively. (4) Prospective stage: Following interim analysis, prospective data collection has been implemented. In addition, a PTS bio-bank network has been created to link clinical data with tumor pathology and molecular analysis.

Results It took 18 months to implement stages 1 to 3 of this model and stage 4 is ongoing. A total of 1,495 tumor cases were captured and diagnosed as one of the 18 primary spine tumor subtypes listed (- Fig. 1). The most prevalent diagnosis was chordoma ( $n=344,23 \%)$. There were 674 females and 821 males with a mean age of $43 \pm 19$ years at the time of surgery. Surgical treatment was performed between 1981 and 2012. The survival at 5 and 10 years postsurgery was 71.9 and $53.3 \%$, respectively, with a median survival of 13 years postsurgery (-Fig. 2 ).

Conclusions To date, this is the largest international collection of PTS. This novel scientific model has not only aggregated a large amount of PTS data, but has also established an international collaborative network of spine oncology centers. The access to large volumes of clinical and bio-bank data will generate further research to guide and enhance the clinical management of PTS. This novel scientific model could be of similar tremendous value if applied to other rare neoplastic conditions.

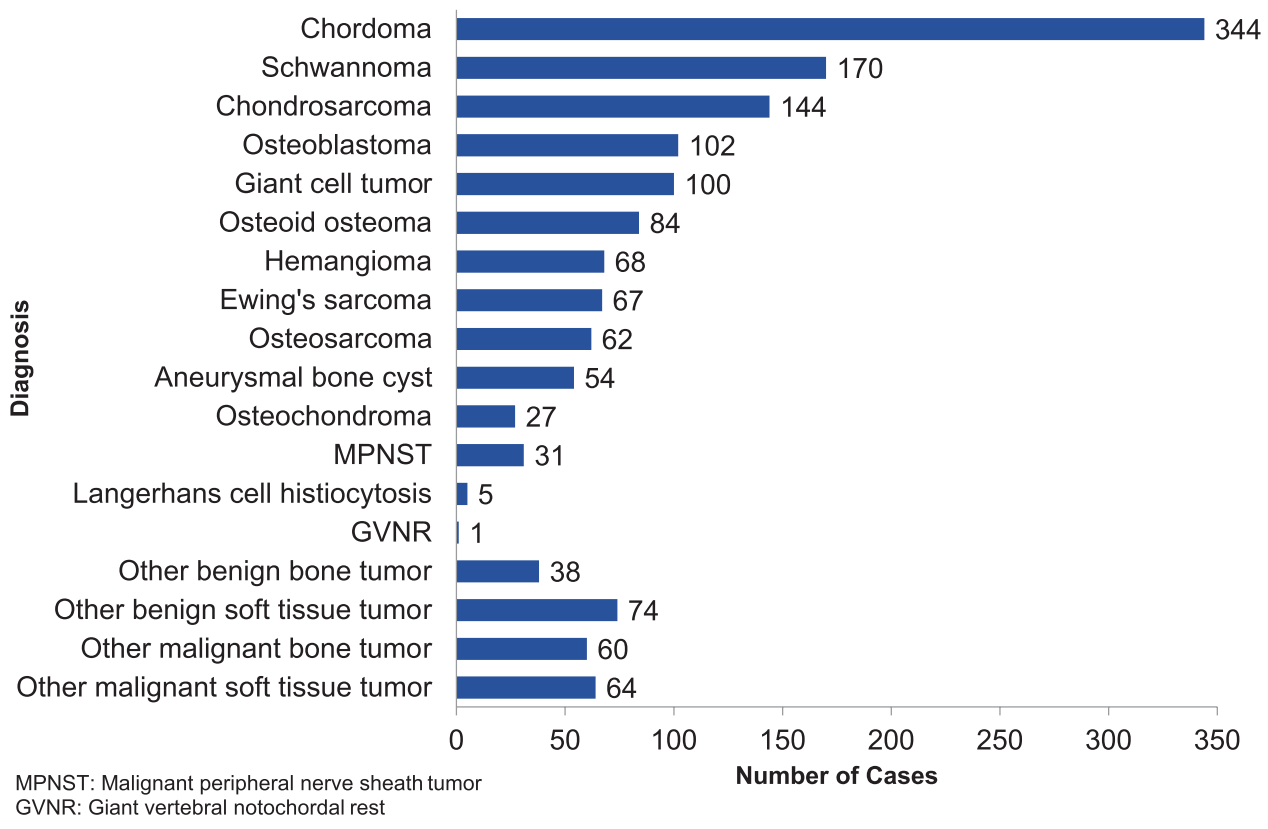

Fig. 1 The diagnosis of 1,495 tumors of the spine cases captured within the AOSpine Knowledge Forum Tumor data set. 


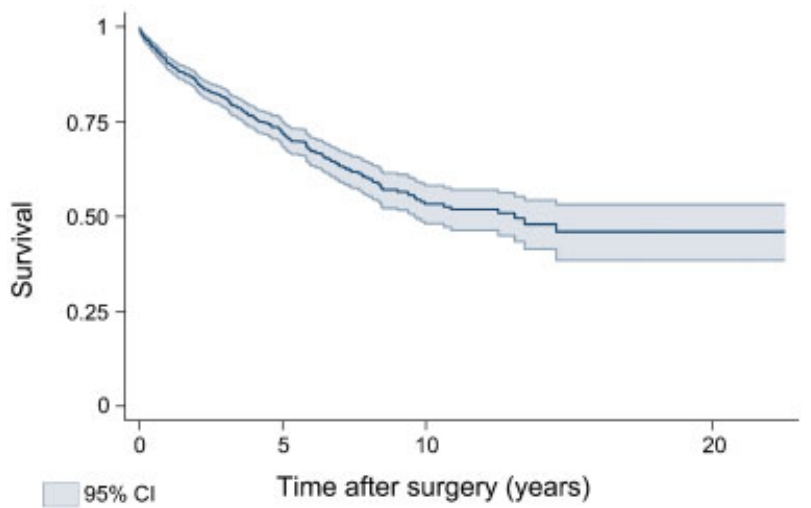

Fig. 2 Survival following surgery.

\section{Funding}

This study was funded by AO Spine International through the AOSpine Knowledge Forum Tumor. Each center received Institute Review Board/Ethics Committee approval.

\section{Reference}

1 Kelley SP, Ashford RU, Rao AS, Dickson RA. Primary bone tumours of the spine: a 42-year survey from the Leeds Regional Bone Tumour Registry. Eur Spine J 2007;16(3):405-409 